\title{
What are the Real Causes of Substrate Inhibition in the Glucuronidation Reaction?
}

\section{Dong Dong, Baojian Wu*}

Department of Pharmacological and Pharmaceutical Sciences, College of Pharmacy, University of Houston, 1441 Moursund Street, Houston, TX 77030, USA

Keywords: Drug metabolism; Glucuronidation; UGTs; Atypical kinetics; Substrate inhibition

UDP-Glucuronosyltransferases (UGTs) are a class of enzymes (please refer to Mackenzie et al. [1] for UGT nomenclature and classification) anchored in the membrane of endoplasmic reticulum. The UGT enzyme catalyzes glucuronidation, a major metabolic pathway in humans, by transferring a glucuronic acid from the cofactor UDPglucuronic acid to the substrate. In vitro characterization has revealed that many human UGT enzymes display substrate inhibition kinetics (i.e., inhibition of enzyme activity at high substrate concentrations) [2]. It is interesting to note that plant UDP-glucosyltransferases (using a similar catalytic mechanism as human UGTs), which are water-soluble, also display substrate inhibition kinetics. For example, plant UGT73C8 and UGT88E1 from Medicago truncatula [3], and UGT78K1 from black soybean (with cyanidin as the substrate) [4].

Although it remains uncertain whether substrate inhibition is operating in vivo, this phenomenon has been observed in cell systems [5,6]. Further, it is hypothesized that substrate inhibition might contribute to regulation in biosynthesis in vivo. For example, the inhibition of UGT78K1 by cyanidin might prevent the cytotoxic effects of high cytosolic cyanidin 3-O-glucoside concentrations [4]. The catalysis and substrate inhibition of dehydroepiandrosterone and androsterone by SULT2A1 may regulate the homeostasis and metabolism of these compounds, and to maintain steroid levels [7].

Understanding substrate inhibition in glucuronidation reactions has been confused by the fact that its occurrence is UGT isoform and (substrate) structure dependent. The isoform-dependency in substrate inhibition is illustrated in Figure 1 for selected substrates. UGT1A1 and $1 \mathrm{~A} 3$ are more prone to the substrate inhibition kinetics, which predominates in the glucuronidation reactions catalyzed by these two isoforms [2]. In contrast, UGT1A7 may be a "typical" enzyme; the glucuronidation reaction catalyzed by this isoform usually obeys the classic Michaelis-Menten kinetics. It is also evident that substrate inhibition is highly structure dependent. For example, although prunetin and genistein are structurally similar isoflavones, glucuronidation of these two compounds by UGT1A1 follows distinct kinetic mechanisms. The former displays Michaelis-Menten and the latter substrate inhibition kinetics [8]. At present, little is known about the structural features for the UGT substrates displaying substrate inhibition.

It has been proposed that the two substrate-binding sites (mechanism I) and the formation of a dead-end ternary complex [E -UDP•S] (mechanism II) might contribute to the substrate inhibition in the glucuronidation reaction [2]. In mechanism I, the two substratebinding sites refer to one reaction and one inhibitory site (so called "effector-binding site"). It is generally favorable that the two substratebinding sites are located in one active site because (a) there is no evidence that an effector-binding site of distant location in relation to the active site exists in the enzymes; and (b) the active sites of UGTs are predicted to be sufficient to accommodate two molecules of a single substrate $[2,9]$. However, the mechanism I is not without question.
According to this mechanism, a small UGT substrate, two molecules of which may be bound in the active site of the enzyme, would have a more tendency to display substrate inhibition. This disagrees with the fact that several big UGT substrates (e.g., traglitazone [Mr $442 \mathrm{Da}$ ] and SN-38 [Mr $392 \mathrm{Da}$ ] for UGT1A1) show substrate inhibition, whereas the much smaller substrates do not (e.g., 4-methylumbelliferone [Mr $176 \mathrm{Da}$ ] for UGT1A1) (Figure 2).

In mechanism II, binding of the substrate to the [E.UDP] complex results in a nonproductive (so called "dead-end") [E•UDP•S] ternary complex, trapping a portion of the enzyme in the inactive form and slowing the catalytic cycle [2]. If this is the working mechanism for substrate inhibition, one may argue that substrate inhibition kinetics should be kept for all substrates that are (highly) structurally related because supposedly they can all bind to the [E•UDP] in a similar fashion. On the contrary, 3-hydroxylflavone displays Michaelis-Menten

Substrate inhibition
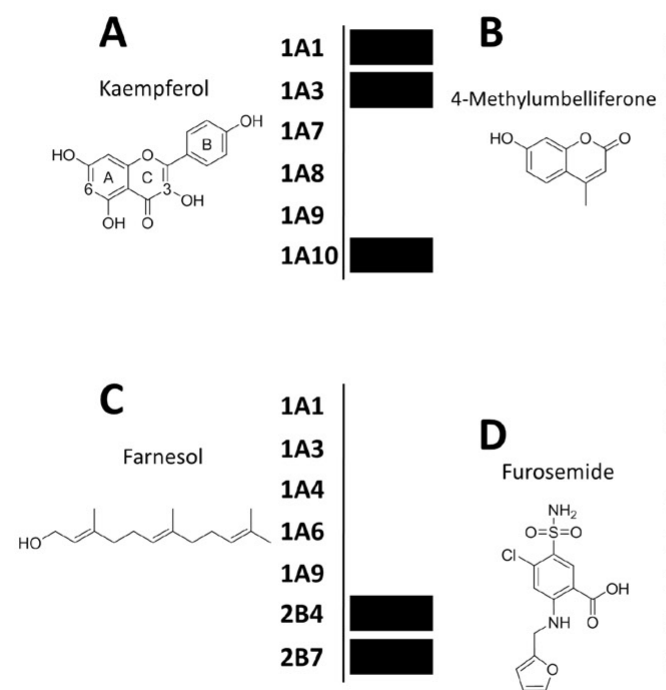

D

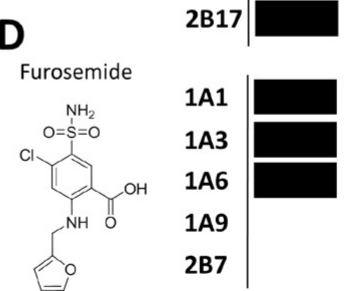

Figure 1: Substrate inhibition is UGT isoform-dependent for the selected substrates (A, kaempferol; B, 4-methylumbelliferone; C, farnesol; D, furosemide)

*Corresponding author: Baojian Wu PhD, 1441 Moursund Street, Department of Pharmacological and Pharmaceutical Sciences, College of Pharmacy, University of Houston, Houston, TX 77030, USA, Tel: (832) 531-1134; Fax:(713)-795-8305; E-mail: bj.wu@hotmail.com

Received April 09, 2012; Accepted April 09, 2012; Published April 16, 2012

Citation: Dong D, Wu B (2012) What are the Real Causes of Substrate Inhibition in the Glucuronidation Reaction? Single Cell Biol 1:e105. doi:10.4172/21689431.1000e105

Copyright: () 2012 Dong D, et al. This is an open-access article distributed under the terms of the Creative Commons Attribution License, which permits unrestricted use, distribution, and reproduction in any medium, provided the original author and source are credited. 
Citation: Dong D, Wu B (2012) What are the Real Causes of Substrate Inhibition in the Glucuronidation Reaction? Single Cell Biol 1:e105. doi:10.4172/2168-9431.1000e105

Page 2 of 3

Michaelis-Menten Kinetics $\quad$ Substrate inhibition Kinetics
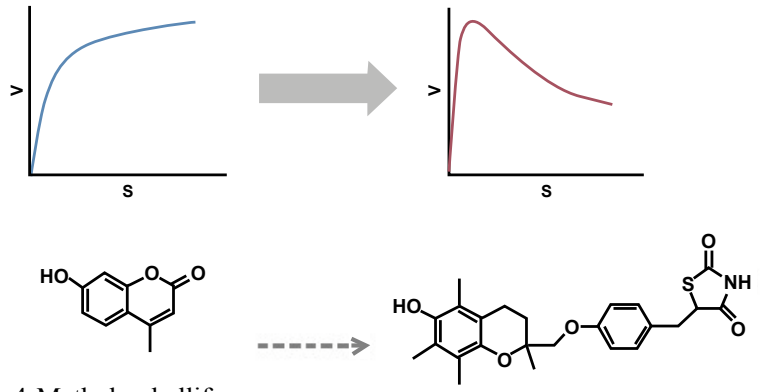

4-Methylumbelliferone
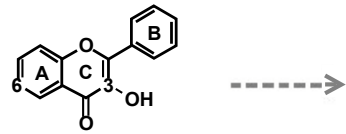

3-Hydroxyflavone

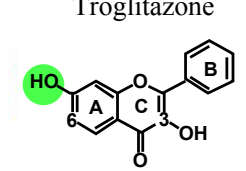

3,7-Dihydroxyflavone

Figure 2: Comparisons of UGT substrates (for UGT1A1) with Michaelis-Menten vs. with substrate inhibition kinetics. Big UGT substrate traglitazone shows substrate inhibition, whereas the much smaller substrate 4-methylumbelliferone demonstrates substrate inhibition. 3-Hydroxyflavone displays Michaelis-Menten kinetics, whereas its analog 3,7-dihydroxyflavone demonstrates substrate inhibition.
A

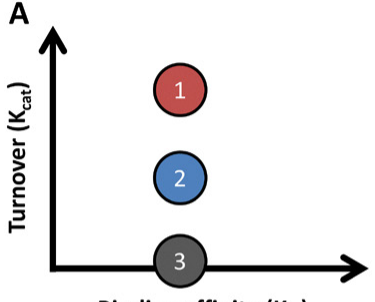

Binding affinity $\left(K_{m}\right)$
E

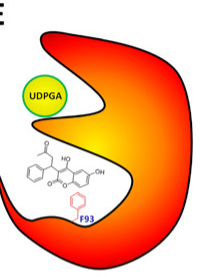

$\mathbf{F}$

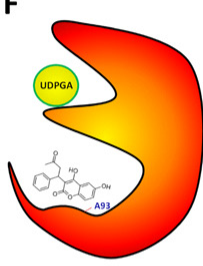

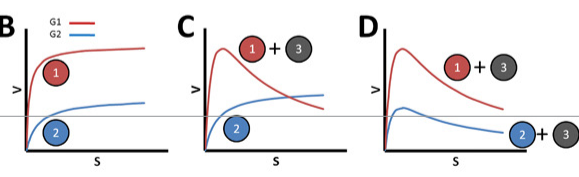

Figure 3: Schematic illustration of the diversity and heterogeneity in substrate-binding modes in UGT reactions for a single substrate. Panel A: Three distinct binding modes are compared with similar binding affinity $(\mathrm{Km})$ but divergent maximal turnover (Kcat or Vmax), red circle 1 denotes active binding mode, blue circle 2 less active binding mode, and gray circle 3 nonproductive binding mode. Panels B-D match the Michaelis-Menten and substrate inhibition kinetics with the distinct binding modes, assuming two glucuronides (G1 and G2) are generated from a substrate. Panels E/F: Single site mutation (F93A) of UGT1A10 changes the Michaelis-Menten kinetics $(E)$ to substrate inhibition kinetics $(F)$, using 6-hydroxywarfarin as an example $[14,15]$. This mutation leads to a nonproductive substrate binding mode that is responsible for the substrate inhibition. This binding mode became nonproductive possibly due to the difficulty in the substrate/glucuronide departure from the protein.

kinetics, whereas its analog 3, 7-dihydroxyflavone demonstrates substrate inhibition (Figure 2). A similar observation is made between prunetin and genistein, two isoflavones. Apparently, this fact is not completely consistent with the mechanism II for substrate inhibition.

Extensive kinetic characterization on the formation of multiple glucuronides from a single substrate and the successful modeling of UGT1A9-mediated 3-O-glucuronidation of flavonols strongly indicate that distinct binding modes are possible in the catalytic domain of UGT protein [10,11]. The different substrate-binding modes are associated with distinct kinetic constants (especially the maximal turnover or $\mathrm{Vmax}$ ) and even distinct kinetic profiles [10]. For example, 7-O-glucuronidation of galangin by UGT1A9 follows MichaelisMenten kinetics $(\mathrm{Km}=0.54 \mu \mathrm{M}$, and $\mathrm{Vmax}=2.56 \mathrm{nmol} / \mathrm{min} / \mathrm{mg}$ protein), whereas 3-O-glucuronidation follows substrate inhibition $(\mathrm{Km}=0.68 \mu \mathrm{M}, \mathrm{Ksi}=7.43 \mu \mathrm{M}$, and $\mathrm{Vmax}=7.41 \mathrm{nmol} / \mathrm{min} / \mathrm{mg}$ protein $)$ [10]. This leads to the hypothesis that the substrate-binding modes in UGT exhibit heterogeneity for a single substrate; some binding modes are active, some are less active (e.g., the modes in which the departure of the substrate/product from the protein is difficult), and some even can be inactive. The substrate inhibition kinetics is resulted from the substrate-binding mode(s) which are nonproductive (or poorly productive). The diversity in substrate-binding modes for UGT reactions is illustrated in Figure 3.A-D. This helps to understand that substrate binding in the UGT active site exhibits flexibility and diversity, allowing the existence of productive and nonproductive modes.

Although the evidence is still lacking, studies might lend support to the hypothesis that substrate inhibition should be associated with a nonproductive binding mode of the substrate in the UGT active site. Please note that this nonproductive binding mode is independent of UDP or UDPGA binding. The F90-M91-V92-F93 amino acid motif (might situate at the entrance of the active site) of UGT1A10 involves in binding the substrates such as hydroxywarfarins and estrogens [1215]. In contrast to that UGT1A10 wild-type displays Michaelis-Menten kinetics, the V92A, F93G, and F93A mutants demonstrate substrate inhibition kinetics. The substrate inhibition displayed by The UGT1A10 mutant (e.g., F93A) might be explained by the induced nonproductive binding mode (see Figure 3. E/F for illustration). Interestingly, this change in the kinetic profile to substrate inhibition induced by single amino acid alteration has also been observed in the sulfotransferase 2A1 (SULT2A1), which mediates the sulfonation reaction, another type of conjugation [16].

In summary, the answers are still open to the question "what are the real causes of substrate inhibition in the glucuronidation reaction?" Explanation is lacking as to why substrate inhibition is UGT isoform and (substrate) structure dependent. A deeper understanding of substrate inhibition will be necessary as the knowledge will promote an alternative functional (and possible regulation) mechanisms of the enzymes, and help to predict glucuronidation at a turnover level. It is without doubt that Journal of Single Cell Genomics \& Proteomics, as an open access journal, could contribute to this field with its published article about protein sciences.

\section{References}

1. Mackenzie PI, Bock KW, Burchell B, Guillemette C, Ikushiro S et al. (2005) Nomenclature update for the mammalian UDP glycosyltransferase (UGT) gene superfamily. Pharmacogenet Genomics 15: 677-685.

2. Wu B (2011) Substrate inhibition kinetics in drug metabolism reactions. Drug Metab Rev 43: 440-456.

3. Modolo LV, Blount JW, Achnine L, Naoumkina MA, Wang X, et al. (2007) A functional genomics approach to (iso)flavonoid glycosylation in the model legume Medicago truncatula. Plant Mol Biol 64: 499-518.

4. Kovinich N, Saleem A, Arnason JT, Miki B (2010) Functional characterization of a UDP-glucose:flavonoid 3-O-glucosyltransferase from the seed coat of black soybean (Glycine max (L.) Merr.). Phytochemistry 71: 1253-1263.

5. Hallifax D, Rawden HC, Hakooz N, Houston JB (2005) Prediction of metabolic clearance using cryopreserved human hepatocytes: kinetic characteristics for five benzodiazepines. Drug Metab Dispos 33: 1852-1858.

6. Sun H, Zhang L, Chow EC, Lin G, Zuo Z, et al. (2008) A catenary model to 
Citation: Dong D, Wu B (2012) What are the Real Causes of Substrate Inhibition in the Glucuronidation Reaction? Single Cell Biol 1:e105. doi:10.4172/2168-9431.1000e105

Page 3 of 3

study transport and conjugation of baicalein, a bioactive flavonoid, in the Caco2 cell monolayer: demonstration of substrate inhibition. J Pharmacol Exp Ther 326: $117-126$.

7. Chang HJ, Shi R, Rehse P, Lin SX (2004) Identifying androsterone (ADT) as a cognate substrate for human dehydroepiandrosterone sulfotransferase (DHEA-ST) important for steroid homeostasis: structure of the enzyme-ADT complex. J Biol Chem 279: 2689-2696.

8. Tang L, Singh R, Liu Z, Hu M (2009) Structure and concentration changes affect characterization of UGT isoform-specific metabolism of isoflavones. Mol Pharm 6: 1466-1482

9. Wu B, Basu S, Meng S, Wang X, Hu M (2011) Regioselective Sulfation and Glucuronidation of Phenolics: Insights into the Structural Basis. Curr Drug Metab 12: 900-916.

10. Wu B, Xu B, Hu M (2011) Regioselective glucuronidation of flavonols by six human UGT1A isoforms. Pharm Res 28: 1905-1918.

11. Wu B, Morrow JK, Singh R, Zhang S, Hu M (2011) Three-dimensional quantitative structure-activity relationship studies on UGT1A9-mediated 3-O-glucuronidation of natural flavonols using a pharmacophore-based comparative molecular field analysis model. J Pharmacol Exp Ther 336: 403413.
12. Xiong Y, Bernardi D, Bratton S, Ward MD, Battaglia E, et al. (2006) Phenylalanine 90 and 93 are localized within the phenol binding site of human UDP-glucuronosyltransferase $1 \mathrm{~A} 10$ as determined by photoaffinity labeling mass spectrometry, and site-directed mutagenesis. Biochemistry 45: 2322 2332.

13. Starlard-Davenport A, Xiong Y, Bratton S, Gallus-Zawada A, Finel M, et al (2007) Phenylalanine(90) and phenylalanine(93) are crucial amino acids within the estrogen binding site of the human UDP-glucuronosyltransferase $1 \mathrm{~A} 10$. Steroids 72: 85-94.

14. Miller GP, Lichti CF, Zielinska AK, Mazur A, Bratton SM, et al. (2008) Identification of hydroxywarfarin binding site in human UDP glucuronosyltransferase 1a10: phenylalanine 90 is crucial for the glucuronidation of 6- and 7-hydroxywarfarin but not 8-hydroxywarfarin. Drug Metab Dispos 36: 2211-2218.

15. Höglund C, Sneitz N, Radominska-Pandya A, Laakonen L, Finel M (2011) Phenylalanine 93 of the human UGT1A10 plays a major role in the interactions of the enzyme with estrogens. Steroids 76: 1465-1473.

16. Lu LY, Hsieh YC, Liu MY, Lin YH, Chen CJ, et al. (2008) Identification and characterization of two amino acids critical for the substrate inhibition of human dehydroepiandrosterone sulfotransferase (SULT2A1). Mol Pharmacol 73: 660668. 